doi: ISSN 0103-5150

Fisioter. Mov., Curitiba, v. 26, n. 4, p. página 873-881, set./dez. 2013

Licenciado sob uma Licença Creative Commons

\title{
Avaliação da qualidade de vida e do tratamento fisioterapêutico em pacientes com cervicalgia crônica
}

\author{
Evaluation of quality of life and the physiotherapy \\ treatment in patients with chronic neck pain
}

\section{Marisa de Carvalho Borges ${ }^{[a]}$, Cláudia dos Santos Borges ${ }^{[b]}$, Ana Graziela Jordão Silva ${ }^{[c]}$, Lúcio Roberto Cançado Castellano $^{[\mathrm{d}]}$, Fabrizio Antônio Gomide Cardoso ${ }^{[\mathrm{e}]}$}

[a] Fisioterapeuta, mestre em Ciências da Saúde pela Universidade Federal do Triângulo Mineiro, Uberaba, MG - Brasil, e-mail: marisa.fisioterapia@yahoo.com.br

[b] Fisioterapeuta, mestre em Clínica Médica pela Universidade Estadual de Campinas (Unicamp) Campinas, SP - Brasil, e-mail: claudinhasb@hotmail.com

[c] Fisioterapeuta, graduada na Universidade Federal do Triângulo Mineiro, Uberaba, MG - Brasil. e-mail: anaga_fisio@hotmail.com

[d] Biomédico, doutor em Medicina Tropical e Infectologia pela Universidade Federal do Triângulo Mineiro (UFTM), professor da Escola Técnica de Saúde da Universidade Federal da Paraíba (UFPB), João Pessoa, PB - Brasil, e-mail: lucio@mednet.com.br

[e] Fisioterapeuta, doutor em Patologia pela Universidade Federal do Triângulo Mineiro (UFTM), professor do Departamento de Ciências Biológicas da UFTM, Uberaba, Minas Gerais, Brasil, e-mail: fabrizio@mednet.com.br

\section{Resumo}

Introdução: Para analisar a efetividade do tratamento fisioterapêutico no paciente com dor crônica cervical, este trabalho teve como objetivo avaliar antes e após o tratamento fisioterapêutico: a qualidade de vida com o instrumento WHOQOL-100 e a melhora na amplitude de movimento usando um goniômetro. Materiais e métodos: Participaram deste estudo 15 pacientes (10 mulheres e 5 homens). 0 nível de significância adotado foi de $\mathrm{p}<0,05$. Resultados: Foi verificada melhora significativa após o tratamento fisioterapêutico no domínio I - físico ( $\mathrm{p}=0,0117$ ); domínio II - psicológico ( $\mathrm{p}=0,004)$; domínio III - nível de independência ( $p$ = 0,0006); domínio IV - relações sociais ( $<$ <,0001); e no domínio V - ambiente ( $p=0,0019)$; e também na amplitude articular dos movimentos de flexão cervical ( $p<0,0001)$, extensão cervical $(p=0,0004)$, inclinação lateral á direita $(\mathrm{p}<0,0001)$ inclinação lateral à esquerda $(\mathrm{p}<0,0001)$, rotação lateral à direita $(\mathrm{p}<0,0001)$ e rotação lateral à esquerda $(\mathrm{p}<0,0001)$. Discussão: Andersen et al. (17) relataram que exercícios 
de alongamento melhoram de forma significativa a amplitude de movimento articular em mulheres com cervicalgia crônica. Chiu et al. (20) observaram melhora na dor crônica cervical e na limitação articular após seis semanas de tratamento com TENS e exercícios. Conclusão: 0 presente estudo conclui que a intervenção fisioterapêutica composta de exercícios de alongamento, técnicas de relaxamento, massagens e eletroterapia foram benéficos para melhorar a qualidade de vida e a flexibilidade nos pacientes com cervicalgia crônica.

Palavras-chave: Fisioterapia. Qualidade de vida. Cervicalgia. Goniometria articular.

\begin{abstract}
Introduction: In order to analyze the effectiveness of the physiotherapeutic treatment in patients with chronic neck pain, this work had as a goal to evaluate the period before and after the physiotherapeutic treatment: quality of life as a WHOQOL-100 tool and the improvement of the range of motion (ROM), using a goniometer. Materials and methods: 15 patients were members of this study (10 women and 5 men). Results: Level of significance adopted was $p<0.05$. It was observed significant improvement after physical therapy in the domain I-physical ( $p=0.0117)$, in domain II - psychological ( $p=0.004)$, the domain III - level of independence $(p=0.0006)$, domain IV - social relationships $(p<0.0001)$ and domain $V-$ environment $(p=0.0019)$ and also in joint range of motion of cervical flexion $(p<$ $0.0001)$, cervical extension ( $p=0.0004)$, right head tilt $(p<0.0001)$, left head tilt $(p<0.0001)$ right lateral rotation $(p<0.0001)$ and left lateral rotation ( $p<0.0001)$. Discussion: Andersen et al. (17) reported that stretching exercises significantly improve the range of motion in women with chronic neck pain. Chiu et al. (20) observed improvement in chronic cervical pain and joint limitation after six weeks of treatment with TENS and exercise. Conclusions: This study concludes that the physioterapeutic intervention composed by stretching exercises, relaxation techniques, massage and electrotherapy were of positive effects for improving the quality of life and flexibility in patients with chronic neck pain.
\end{abstract}

Keywords: Physiotherapy. Quality of life. Neck pain. Arthrometry articular.

\section{Introdução}

A cervicalgia crônica é uma síndrome caracterizada por dor e limitação na amplitude de movimento da região cervical, que causa desde pequenos desconfortos até dores intensas ou, até mesmo, incapacitantes (1). Pode acometer $30 \%$ da população adulta em alguma fase da vida, e a maior incidência é no sexo feminino (2).

A queixa de dor na região cervical é referida pelos pacientes como sendo responsável pela redução na qualidade de vida (3), provocando dramática mudança no estilo de vida, dependência de medicamentos, depressão, isolamento social, dificuldades no trabalho e alterações emocionais (4).

A fisioterapia pode desempenhar um papel importante no tratamento do paciente com dor crônica cervical, pois busca diminuir a dor, recuperar a mobilidade e fortalecer a musculatura, proporcionando, dessa forma, melhora na qualidade de vida $(5,6)$.

Instrumentos sobre qualidade de vida são úteis para avaliar a efetividade do tratamento fisioterapêutico, levando-se em conta as repercussões da terapia no paciente como um todo e não só em um grupo muscular específico (7).

Ao considerar a qualidade de vida e introduzir a sua mensuração como critério para avaliar a efetividade do tratamento fisioterapêutico, o profissional passa a entender a opinião do paciente na planificação terapêutica, levando-se em conta as repercussões da terapia no paciente como um todo. A análise desses dados é de fundamental importância, não só para mudar a estratégia terapêutica em um determinado caso como também para que outros pacientes possam se beneficiar dessas observações.

Para analisar a efetividade do tratamento fisioterapêutico no paciente com dor crônica cervical, este trabalho teve como objetivo avaliar antes e após o tratamento fisioterapêutico: a qualidade de vida com o instrumento WHOQOL-100 e a melhora na amplitude de movimento articular (ADM) com a utilização de um goniômetro.

\section{Materiais e métodos}

0 presente estudo de natureza descritivo-analítica foi desenvolvido no Centro de Reabilitação Professor 
Dr. Fausto da Cunha Oliveira, na Universidade Federal do Triângulo Mineiro, na cidade de Uberaba (MG).

O projeto foi aprovado pelo Comitê de Ética em Pesquisa da Universidade Federal do Triângulo Mineiro (Parecer n. 1208). Todos os sujeitos foram orientados quanto aos objetivos do estudo e quanto ao caráter voluntário e confidencial de sua participação. A concordância foi registrada com a assinatura do termo de consentimento livre após esclarecimento.

Participaram deste estudo 15 pacientes portadores de dores crônicas na coluna cervical. A idade mínima foi de 43 anos e a máxima de 83 anos, com média de idade de 61,3 anos. Desses, dez (66,6\%) pacientes eram do sexo feminino e cinco $(33,4 \%)$ do sexo masculino.

0 tratamento fisioterapêutico foi realizado durante 12 semanas consecutivas, segundas, quartas e sextas-feiras, perfazendo um total de 36 sessões, com duração de 110 minutos cada uma. No protocolo terapêutico organizado pelos pesquisadores os pacientes realizaram os tratamentos relacionados a seguir.

- TENS: foi utilizada a corrente Tens convencional com frequência (R) de $100 \mathrm{~Hz}$, duração do pulso de $150 \mu$ s, aplicado por 45 minutos e intensidade ajustada de acordo com a sensibilidade do paciente. Foram utilizados quatro eletrodos de $15 \mathrm{~cm}^{2}(5 \mathrm{~cm} \times 3 \mathrm{~cm})$ de borracha siliconada e carbonada, posicionados em paralelo entre si na região cervical, gel condutor e fita adesiva para fixá-los e um eletroestimulador KW modelo Kinesis New Microcontrolled ${ }^{\circledR}$, novo e devidamente calibrado pelo fabricante.

- Radiação infravermelha: a lâmpada de 150 W da Philips foi inicialmente posicionada a uma distância de $60 \mathrm{~cm}$ do paciente e a intensidade foi alterada, mudando a distância entre a lâmpada e a coluna cervical. 0 tratamento ocorreu por um período de 10 a 20 minutos. Todos os pacientes receberam o tratamento com a Tens (18 sessões) e com a radiação infravermelha (18 sessões) em dias alternados.

- Alongamentos: foram realizados exercícios de alongamento para os músculos: levantador da escápula, trapézio, esternocleidomastoideo, esplênio da cabeça e do pescoço, semiespinal da cabeça e do pescoço, longuíssimo da cabeça e do pescoço, espinal da cabeça e do pescoço, platisma, romboides, latíssimo do dorso, deltoide anterior, deltoide posterior, peitoral maior e menor, tríceps braquial, infraespinal, redondo maior e menor, serrátil anterior, semiespinal do pescoço e do tórax, espinal do pescoço e do tórax, longuíssimo do pescoço e do tórax, iliocostal do pescoço e do tórax, rotadores, bíceps braquial, braquial, braquiorradial. Os alongamentos eram realizados em uma sala ampla, com música, e os pacientes eram incentivados a fazê-los também em casa.

- Massagem terapêutica: a massagem era realizada na região torácica e cervical com o paciente deitado em decúbito ventral, com a cabeça virada para um dos lados e uma almofada sob o abdômen do paciente. As técnicas de massagem realizadas foram: deslizamento para toda a região das costas, deslizamento com os punhos no pescoço e no ombro, deslizamento com o polegar na escápula, compressão na região superior do ombro, deslizamento com os punhos na região torácica, amassamento nos músculos posterolaterais do pescoço, compressão na região do ombro e a técnica neuromuscular para o elevador da escápula, o trapézio e o supraespinhoso.

Os critérios para inclusão na pesquisa foram: idade superior a 20 anos, nenhum tratamento fisioterapêutico prévio, interesse em participar do estudo, disponibilidade para responder às questões sobre qualidade de vida e também para comparecer ao ambulatório nos dias e horários definidos pelos pesquisadores.

Os critérios para exclusão da pesquisa foram: pacientes com dor cervical decorrente de trauma agudo, pacientes com ferimentos abertos na região cervical ou que apresentassem desconfortos durante o tratamento que o tornassem inviável.

O WHOQOL-100 foi entregue a cada paciente antes e após o tratamento fisioterapêutico. Esse instrumento possui seis domínios (psicológico, físico, nível de independência, relações sociais, ambiente e espiritualidade), constituídos por facetas que são avaliadas por quatro questões (Quadro 1). Assim, o instrumento é composto por 24 facetas específicas e uma faceta geral que inclui questões de avaliação global de qualidade de vida. As respostas para as questões do WHOQOL são dadas em uma escala do tipo Likert (8). Para quantificar limitações, foi realizada a mensuração da ADM cervical utilizando um goniômetro universal ( $\mathrm{Carci}^{\circledR}{ }^{\circledR}$, Indústria e Comércio de Aparelhos Cirúrgicos e Ortopédicos Ltda, Brasil), 
Quadro 1 - Domínios e facetas do Instrumento de Avaliação da Qualidade de Vida da OMS (WHOQOL)

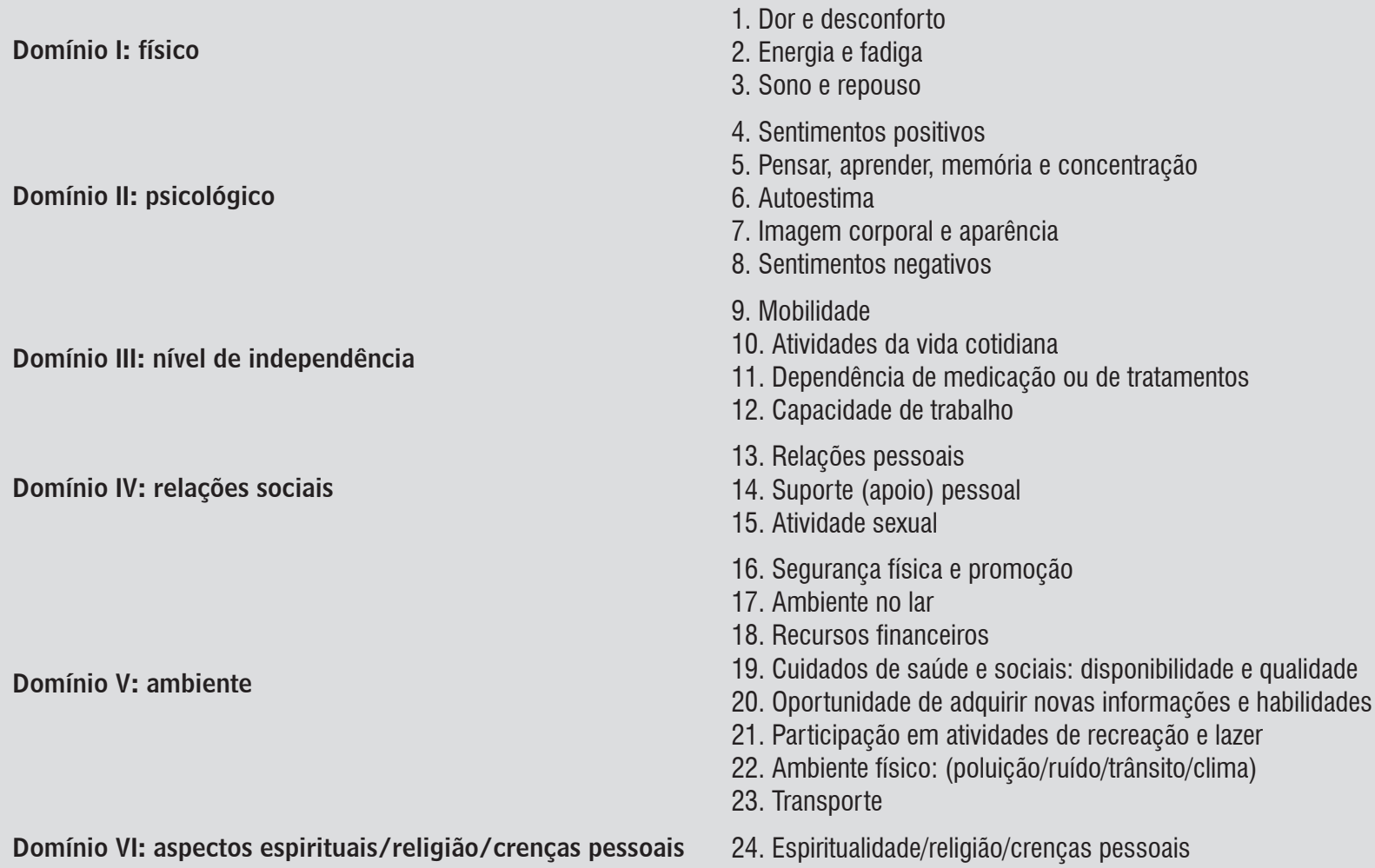

1. Dor e desconforto

2. Energia e fadiga

3. Sono e repouso

4. Sentimentos positivos

5. Pensar, aprender, memória e concentração

6. Autoestima

7. Imagem corporal e aparência

8. Sentimentos negativos

9. Mobilidade

10. Atividades da vida cotidiana

11. Dependência de medicação ou de tratamentos

12. Capacidade de trabalho

13. Relações pessoais

14. Suporte (apoio) pessoal

15. Atividade sexual

16. Segurança física e promoção

17. Ambiente no lar

18. Recursos financeiros

19. Cuidados de saúde e sociais: disponibilidade e qualidade

20. Oportunidade de adquirir novas informações e habilidades

21. Participação em atividades de recreação e lazer

22. Ambiente físico: (poluição/ruído/trânsito/clima)

23. Transporte

24. Espiritualidade/religião/crenças pessoais

com escala de medida de dois em dois graus. A mensuração da ADM cervical foi realizada antes e após o tratamento fisioterapêutico e os movimentos avaliados na região cervical foram: flexão cervical $\left(0^{\circ}-\right.$ $\left.65^{\circ}\right)$, extensão cervical $\left(0^{\circ}-50^{\circ}\right)$, inclinação lateral à direita $\left(0^{\circ}-40^{\circ}\right)$, inclinação lateral à esquerda $\left(0^{\circ}-\right.$ $\left.40^{\circ}\right)$, rotação lateral à direita $\left(0^{\circ}-55^{\circ}\right)$ e rotação lateral à esquerda $\left(0^{\circ}-55^{\circ}\right)$.

$\mathrm{Na}$ análise estatística os questionários foram pontuados de acordo com a sintaxe de análise do Whoqol 100, pelo programa SPSS, de acordo com o recomendado pela Organização Mundial de Saúde, e disponibilizado para a versão nacional em <http:// www.ufrgs.br/Psiq/whoqol85.html>. A partir daí os dados foram testados quanto à normalidade pelo teste de Kolmogorov-Smirnov e, posteriormente, comparados entre si pelo teste de "t pareado". Foram consideradas estatisticamente significativas as diferenças com $\mathrm{p}<0,05$.

Os dados da goniometria também foram testados quanto à normalidade pelo teste de KolmogorovSmirnov e posteriormente comparados pelo teste de "t pareado". O nível de significância adotado foi de $\mathrm{p}<0,05$. Foram utilizados os softwares Microsoft Excel 2007, GraphPad Prism 5.0 e SPSS 16.0.

\section{Resultados}

O instrumento sobre qualidade de vida apresentou resultados positivos significantes nos seguintes domínios: domínio 1 - físico ( $\mathrm{p}=0,0117$ ); domínio 2 - psicológico $(\mathrm{p}=0,004)$; domínio 3 - nível de independência $(\mathrm{p}=0,0006)$; domínio 4 - relações sociais ( $p<0,0001)$; domínio 5 - ambiente $(\mathrm{p}=0,0019)$ (Figura 1, A-F). 0 mesmo não ocorreu com o domínio 6 - aspectos espirituais/religião/crenças pessoais ( $\mathrm{p}=0,7921)$.

Os resultados também mostraram significância estatística nas seguintes facetas: dor e desconforto $(p=0,0214)$, energia e fadiga $(p=0,0078)$, sentimentos positivos ( $p=0,0206)$, imagem corporal e aparência $(p=0,0418)$, sentimentos negativos $(p=0,0266)$, atividade da vida cotidiana ( $\mathrm{p}=00182)$, dependência de medicação ou de tratamentos ( $\mathrm{p}<0,0001)$, capacidade de trabalho $(p=0,0025)$, segurança física e proteção $(p=0,0359)$ e ambiente físico $(p=0,0061)$ (Tabela 1$)$. 

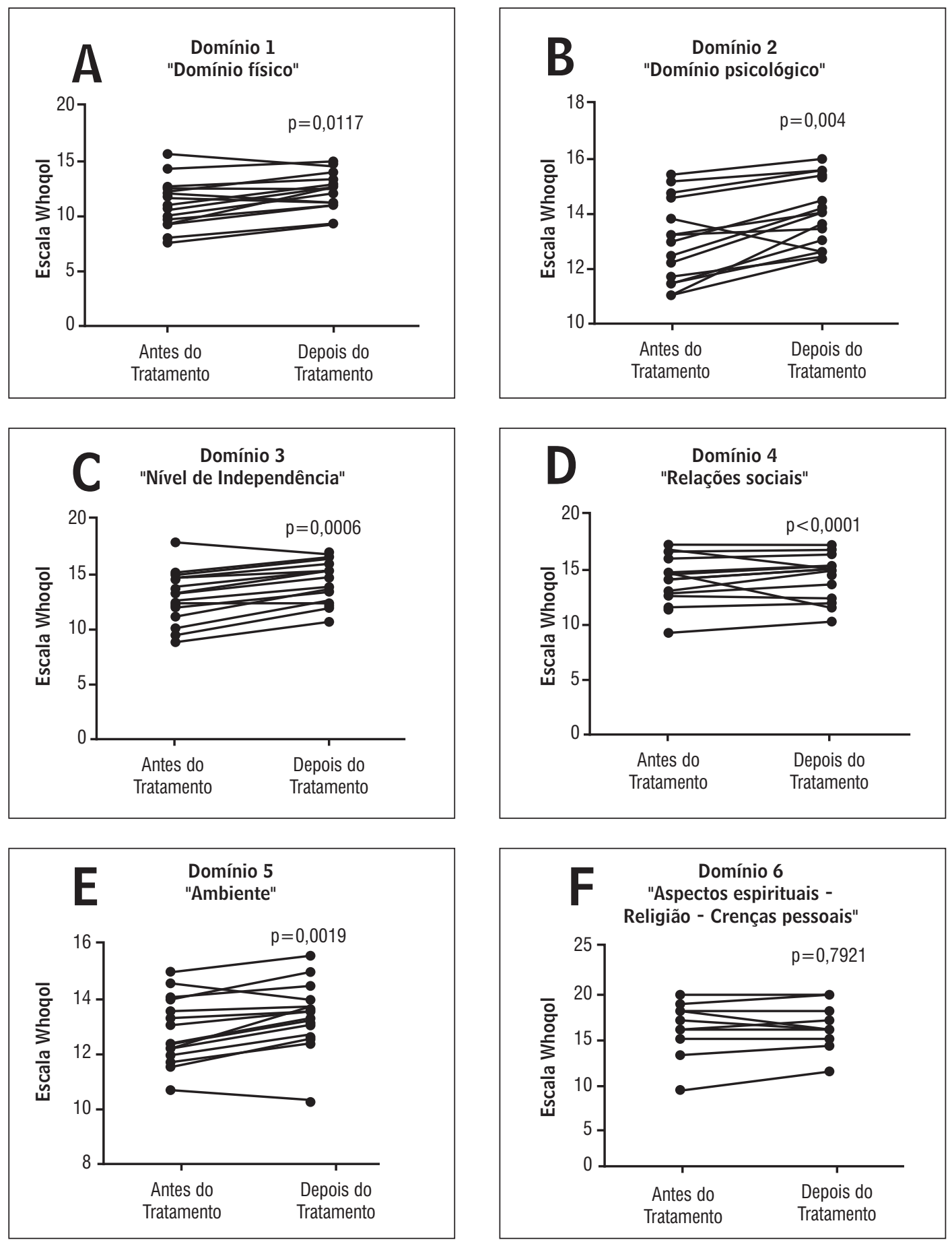

Figura 1 - Valores dos domínios antes e após o tratamento fisioterapêutico Fonte: Dados da pesquisa.

Foi observado que o tratamento aplicado acarretou melhora significativa nos movimentos de flexão cervical, extensão cervical, inclinação lateral à direita, inclinação lateral à esquerda, rotação lateral à direita e rotação lateral à esquerda, mostrados na Tabela 2 .

\section{Discussão}

A dor crônica é uma das principais causas de incapacidade física e funcional, pois compromete as atividades de vida diária, de lazer, as relações sociais, 
Tabela 1 - Valores da média e desvio padrão das "facetas e domínios" antes e após o tratamento fisioterapêutico e os valores de "p"

\begin{tabular}{|c|c|c|c|c|}
\hline \multicolumn{2}{|c|}{ Facetas e domínios } & \multirow{2}{*}{$\begin{array}{l}\begin{array}{l}\text { Antes do tto } \\
\text { Média } \pm \text { DP }\end{array} \\
15,60 \pm 1,99\end{array}$} & \multirow{2}{*}{$\begin{array}{c}\begin{array}{c}\text { Depois do tto } \\
\text { Média } \pm \text { DP }\end{array} \\
14,13 \pm 1,60\end{array}$} & \multirow{2}{*}{$\begin{array}{c}\begin{array}{c}\text { Valor de } \\
\text { "p" }\end{array} \\
0,0214\end{array}$} \\
\hline Faceta 1 & Dor e desconforto & & & \\
\hline Faceta 2 & Energia e fadiga & $11,80 \pm 2,62$ & $13,20 \pm 2,11$ & 0,0078 \\
\hline Faceta 3 & Sono e repouso & $13,13 \pm 3,76$ & $13,60 \pm 3,44$ & 0,265 \\
\hline Faceta 4 & Sentimentos positivos & $12,20 \pm 1,57$ & $13,40 \pm 1,64$ & 0,0206 \\
\hline Faceta 5 & Pensar, aprender, memória e concentração & $13,00 \pm 2,88$ & $13,47 \pm 2,26$ & 0,2199 \\
\hline Faceta 6 & Autoestima & $14,33 \pm 2,50$ & $15,47 \pm 2,56$ & 0,0799 \\
\hline Faceta 7 & Imagem corporal e aparência & $15,07 \pm 3,17$ & $16,20 \pm 2,96$ & 0,0418 \\
\hline Faceta 8 & Sentimentos negativos & $13,87 \pm 3,40$ & $12,80 \pm 2,65$ & 0,0266 \\
\hline Faceta 9 & Mobilidade & $13,13 \pm 2,00$ & $13,33 \pm 1,99$ & 0,4243 \\
\hline Faceta 10 & Atividades da vida cotidiana & $11,73 \pm 2,60$ & $13,13 \pm 2,00$ & 0,0182 \\
\hline Faceta 11 & Dependência de medicação ou de tratamentos & $12,20 \pm 3,90$ & $9,93 \pm 2,99$ & $<0,0001$ \\
\hline Faceta 12 & Capacidade de trabalho & $13,00 \pm 3,21$ & $14,60 \pm 1,96$ & 0,0025 \\
\hline Faceta 13 & Relações pessoais & $15,60 \pm 2,69$ & $15,40 \pm 2,77$ & 0,5816 \\
\hline Faceta 14 & Suporte (apoio) social & $13,07 \pm 2,46$ & $13,40 \pm 2,38$ & 0,4849 \\
\hline Faceta 15 & Atividade sexual & $12,87 \pm 2,85$ & $13,00 \pm 2,93$ & 0,7284 \\
\hline Faceta 16 & Segurança física e proteção & $11,13 \pm 1,85$ & $11,80 \pm 1,61$ & 0,0359 \\
\hline Faceta 17 & Ambiente no lar & $14,93 \pm 2,49$ & $15,40 \pm 2,90$ & 0,1689 \\
\hline Faceta 18 & Recursos financeiros & $11,07 \pm 1,58$ & $11,87 \pm 1,92$ & 0,0824 \\
\hline Faceta 19 & $\begin{array}{l}\text { Cuidados de saúde e sociais: } \\
\text { disponibilidade e qualidade }\end{array}$ & $14,07 \pm 2,40$ & $14,13 \pm 2,53$ & 0,86 \\
\hline Faceta 20 & $\begin{array}{l}\text { Oportunidades de adquirir novas informações e } \\
\text { habilidades }\end{array}$ & $13,00 \pm 2,14$ & $13,33 \pm 1,80$ & 0,3535 \\
\hline Faceta 21 & Participação em atividades de recreação e lazer & $10,07 \pm 2,76$ & $10,73 \pm 2,99$ & 0,1458 \\
\hline Faceta 22 & Ambiente físico (poluição, ruído, trânsito, clima) & $12,67 \pm 1,35$ & $13,73 \pm 1,67$ & 0,0061 \\
\hline Faceta 23 & Transporte & $13,67 \pm 2,89$ & $13,67 \pm 2,41$ & 1 \\
\hline Faceta 24 & Espiritualidade, religião, crenças pessoais & $16,07 \pm 2,60$ & $16,13 \pm 2,20$ & 0,7921 \\
\hline Domínio 1 & Domínio físico & $11,11 \pm 2,22$ & $12,22 \pm 1,73$ & 0,0117 \\
\hline Domínio 2 & Domínio psicológico & $12,95 \pm 1,54$ & $13,95 \pm 1,25$ & 0,004 \\
\hline Domínio 3 & Nível de independência & $12,42 \pm 2,33$ & $13,78 \pm 1,82$ & 0,0006 \\
\hline Domínio 4 & Relações sociais & $13,84 \pm 2,06$ & $13,93 \pm 1,93$ & $<0,0001$ \\
\hline Domínio 5 & Ambiente & $12,58 \pm 1,16$ & $13,08 \pm 1,17$ & 0,0019 \\
\hline Domínio 6 & Aspectos espirituais - religião, crenças pessoais & $16,07 \pm 2,60$ & $16,13 \pm 2,20$ & 0,7921 \\
\hline
\end{tabular}

Fonte: Dados da pesquisa.

familiares e laborativas, comprometendo de forma significativa a qualidade de vida em ambos os sexos (9). Segundo Leboeuf-Yde et al. (10), a cervicalgia crônica é mais frequente em pacientes do sexo feminino. Esses dados corroboram com nossos resultados, pois no presente estudo $66,6 \%$ dos pacientes tratados eram mulheres.
A fisioterapia atua no tratamento da cervicalgia crônica, dispondo de recursos eletroterapêuticos e programas de exercício que visam melhorar o condicionamento muscular, a flexibilidade e o alívio sintomático da dor. Esses benefícios proporcionam maior disposição e facilidade para a realização de atividades cotidianas, melhorando a qualidade de vida $(11,12)$. 
Tabela 2 - Valores de média e desvio padrão para todos os parâmetros avaliados, antes e após tratamento fisioterapêutico

\begin{tabular}{|c|c|c|c|}
\hline \multicolumn{4}{|c|}{ FLEXÃO DA REGIÃO CERVICAL } \\
\hline & Antes do tratamento & Após o tratamento & valor de "p" \\
\hline Média & 63,00 & 98,00 & \\
\hline Desvio padrão & 14,12 & 15,45 & $p<0,0001$ \\
\hline Máximo & 100,00 & 120,00 & \\
\hline Mínimo & 45,00 & 75,00 & \\
\hline \multicolumn{4}{|c|}{ EXTENSÃO DA REGIÃO CERVICAL } \\
\hline & Antes do tratamento & Após o tratamento & valor de "p" \\
\hline Média & 54,67 & 76,00 & \\
\hline Desvio padrão & 14,45 & 15,38 & $p=0,0004$ \\
\hline Máximo & 80,00 & 120,00 & \\
\hline Mínimo & 35,00 & 55,00 & \\
\hline \multicolumn{4}{|c|}{ INCLINAÇÃO LATERAL À DIREITA } \\
\hline & Antes do tratamento & Após o tratamento & valor de "p" \\
\hline Média & 32,67 & 51,00 & \\
\hline Desvio padrão & 7,99 & 15,26 & $p<0,0001$ \\
\hline Máximo & 45,00 & 80,00 & \\
\hline Mínimo & 15,00 & 25,00 & \\
\hline \multicolumn{4}{|c|}{ INCLINAÇÃO LATERAL À ESQUERDA } \\
\hline & Antes do tratamento & Após o tratamento & valor de "p" \\
\hline Média & 37,67 & 54,67 & \\
\hline Desvio padrão & 9,80 & 9,54 & $p<0,0001$ \\
\hline Máximo & 50,00 & 70,00 & \\
\hline Mínimo & 25,00 & 40,00 & \\
\hline \multicolumn{4}{|c|}{ ROTAÇÃO LATERAL À DIREITA } \\
\hline & Antes do tratamento & Após o tratamento & valor de "p" \\
\hline Média & 46,00 & 65,67 & \\
\hline Desvio padrão & 10,39 & 11,00 & $p<0,0001$ \\
\hline Máximo & 70,00 & 85,00 & \\
\hline Mínimo & 30,00 & 50,00 & \\
\hline \multicolumn{4}{|c|}{ ROTAÇÃO LATERAL À ESQUERDA } \\
\hline & Antes do tratamento & Após o tratamento & valor de "p" \\
\hline Média & 43,33 & 61,67 & \\
\hline Desvio padrão & 10,29 & 11,13 & $p<0,0001$ \\
\hline Máximo & 65,00 & 90,00 & \\
\hline Mínimo & 25,00 & 50,00 & \\
\hline
\end{tabular}

Fonte: Dados da pesquisa.

Para Mason et al. (13), instrumentos sobre qualidade de vida avaliam o impacto físico e psicossocial que as enfermidades, disfunções ou incapacidades causam nas pessoas acometidas.
Os resultados do estudo de Brasil et al. (14), na avaliação da qualidade de vida do portador de dor crônica em tratamento com acupuntura, indicaram que as dimensões que abordam o impacto do nível 
de dor e da saúde física, bem como o reflexo das condições emocionais no desempenho das atividades diárias e/ou profissionais, são as que necessitam de intervenção profissional imediata para melhorar a qualidade de vida desses indivíduos.

As queixas de dor crônica estão relacionadas à redução da capacidade funcional (15), pois na realização das tarefas cotidianas, ocupacionais e recreativas é necessária uma amplitude de movimentos sem restrições e sem dor. A falta de flexibilidade gera diminuição na capacidade física de indivíduos com cervicalgia, causando restrições e lentificação para a realização das atividades diárias, comprometendo seriamente a qualidade de vida (16).

Andersen et al. (17) relataram que exercícios de alongamento melhoram de forma significativa a amplitude de movimento articular em mulheres com cervicalgia crônica. Ylinen et al. (18) observaram que a prática de exercícios de alongamento associada à terapia manual por quatro semanas pode diminuir a dor cervical e a limitação articular em 52\%.

Outro método de tratamento não farmacológico bastante utilizado é a eletroterapia. Na prática clínica, quando utilizada como coadjuvante, apresenta resultados satisfatórios, atenuando a dor e melhorando a tensão muscular em pacientes com dor cervical (19). Chiu et al. (20) observaram melhora na dor crônica cervical e na limitação articular após seis semanas de tratamento com TENS e exercícios. Em sua pesquisa, Durmus et al. (21) avaliaram os efeitos da estimulação elétrica e do ultrassom em pacientes com lombalgia crônica, e observaram que o tratamento foi eficaz na melhora da dor, no fortalecimento muscular e na qualidade de vida.

\section{Conclusão}

No presente estudo foi constatado que após o tratamento com a Tens, a radiação infravermelha, os exercícios e a massagem terapêutica, os pacientes com cervicalgia crônica apresentaram resultados satisfatórios nos domínios de qualidade de vida, com maior destaque para o domínio físico, seguido pelo psicológico, independência e social. Antes do tratamento fisioterapêutico, os pacientes estavam apáticos e irritados e com uma visão pessimista em relação à própria saúde. Esses dados mostram que a utilização do instrumento de qualidade de vida foi importante, pois forneceu impressões pessoais de cada paciente, contribuindo para que os profissionais identificassem fatores que pudessem agravar ou diminuir a intensidade dos sintomas. Também possibilitou a avaliação da efetividade do tratamento fisioterapêutico nos pacientes como um todo e não apenas avaliando a melhora da dor em um grupo muscular específico.

\section{Referências}

1. Cassidy JD, Côté P. Is it time for a population health approach to neck pain? J Manipulative Physiol Ther. 2008;31(6):442-6.

2. Strine TW, Hootman JM. US national prevalence and correlates of low back and neck pain among adults. Arthritis Rheum. 2007;57(4):656-65.

3. Barnes PM, Bloom B, Nahin RL. Complementary and alternative medicine use among adults and children: United States, 2007. Natl Health Stat Report. 2008;(12):1-23.

4. Wade JB, Price DD, Hamer RM, Schwartz SM, Hart RP. An emotional component analysis of chronic pain. Pain. 1990;40(3):303-10.

5. Andersen LL, Kjaer M, Søgaard K, Hansen L, Kryger AI, Sjøgaard G. Effect of two contrasting types of physical exercise on chronic neck muscle pain. Arthritis Rheum. 2008;59(1):84-91.

6. Andersen LL, Andersen JL, Suetta C, Kjaer M, Søgaard K, Sjøgaard G. Effect of contrasting physical exercise interventions on rapid force capacity of chronically painful muscles. J Appl Physiol. 2009;107(5):1413-9.

7. Obremskey WT, Brown O, Driver R, Dirschl DR. Comparison of SF-36 and Short Musculoskeletal Functional Assessment in recovery from fixation of unstable ankle fractures. Orthopedics. 2007;30(2):145-51.

8. Fleck MPA, Louzada S, Xavier M, Chamovich E, Vieira G, Santos L, et al. Desenvolvimento da versão em português do instrumento de avaliação da qualidade de vida da OMS (WHOQOL-100). Rev Bras Psiquiatr. 1999;21(1):19-28.

9. Schmitt MA, van Meeteren NL, de Wijer A, van Genderen FR, van der Graaf Y, Helders PJ. Patients with chronic whiplash-associated disorders: relationship between clinical and psychological factors and functional health status. Am J Phys Med Rehabil. 2009;88(3):231-8. 
10. Leboeuf-Yde C, Nielsen J, Kyvik KO, Fejer R, Hartvigsen J. Pain in the lumbar, thoracic or cervical regions: do age and gender matter? A population-based study of 34,902 Danish twins 20-71 years of age. BMC Musculoskelet Disord. 2009;10:39.

11. von Trott P, Wiedemann AM, Lüdtke R, Reishauer A, Willich SN, Witt CM. Qigong and exercise therapy for elderly patients with chronic neck pain (QIBANE): a randomized controlled study. J Pain. 2009;10(5):501-8.

12. Gross A, Miller J, D'Sylva J, Burnie SJ, Goldsmith $\mathrm{CH}$, Graham N, et al. Manipulation or mobilisation for neck pain: a Cochrane Review. Man Ther. 2010; 15(4):315-33.

13. Mason VL, Skevington SM, Osborn M. A measure for quality of life assessment in chronic pain: preliminary properties of the WHOQOL-pain. J Behav Med. 2009;32(2):162-73.

14. Brasil VV, Zatta LT, Cordeiro JABL, Silva AMTC, Zatta DT, Barbosa MA. Qualidade de vida de portadores de dores crônicas em tratamento com acupuntura Rev eletrônica enferm. 2008;10(2):383-94.

15. Capela C, Marques AP, Assumpção A, Sauer JF, Cavalcante $A B$, Chalot $S D$. Associação da qualidade de vida com dor, ansiedade e depressão. Fisioter Pesq. 2009;16 (3):263-8.

16. Cunha AC, Burke TN, França FJ, Marques AP. Effect of global posture reeducation and of static stretching on pain, range of motion, and quality of life in women with chronic neck pain: a randomized clinical trial. Clinics. 2008;63(6):763-70.

17. AndersenLL,Kjaer M,Andersen CH,HansenPB,ZebisMK, Hansen $\mathrm{K}$, et al. Muscle activation during selected strength exercises in women with chronic neck muscle pain. Phys Ther. 2008;88(6):703-11.

18. Ylinen J, Kautiainen H, Wirén K, Häkkinen A. Stretching exercises $v s$ manual therapy in treatment of chronic neck pain: a randomized, controlled crossover trial. J Rehabil Med. 2007;39(2):126-32.

19. Kroeling P, Gross AR, Goldsmith CH; Cervical Overview Group. A Cochrane review of electrotherapy for mechanical neck disorders. Spine. 2005;1,30(21):E641-8.

20. Chiu TT, Hui-Chan CW, Chein G. A randomized clinical trial of TENS and exercise for patients with chronic neck pain. Clin Rehabil. 2005;19(8):850-60.
21. Durmus D, Durmaz Y, Canturk F. Effects of therapeutic ultrasound and electrical stimulation program on pain, trunk muscle strength, disability, walking performance, quality of life, and depression in patients with low back pain: a randomized-controlled trial. Rheumatol Int. 2010;30(7):901-10.

Recebido: 10/03/2013

Received: 03/10/2013

Aprovado: 09/10/2013

Approved: 10/09/2013 